\title{
Recognition of ribosomal protein L11 by the protein trimethyltransferase PrmA
}

\section{Hasan Demirci, Steven T Gregory, Albert E Dahlberg and Gerwald Jogl*}

Department of Molecular Biology, Cell Biology and Biochemistry, Brown University, Providence, RI, USA

Bacterial ribosomal protein $L 11$ is post-translationally trimethylated at multiple residues by a single methyltransferase, PrmA. Here, we describe four structures of PrmA from the extreme thermophile Thermus thermophilus. Two apo-PrmA structures at 1.59 and $2.3 \AA$ resolution and a third with bound cofactor $S$-adenosyl-L-methionine at $1.75 \AA$ each exhibit distinct relative positions of the substrate recognition and catalytic domains, revealing how PrmA can position the L11 substrate for multiple, consecutive side-chain methylation reactions. The fourth structure, the PrmA-L11 enzyme-substrate complex at $2.4 \AA$ resolution, illustrates the highly specific interaction of the $\mathrm{N}$-terminal domain with its substrate and places Lys39 in the PrmA active site. The presence of a unique flexible loop in the cofactor-binding site suggests how exchange of AdoMet with the reaction product $S$-adenosyl-t-homocysteine can occur without necessitating the dissociation of PrmA from L11. Finally, the mode of interaction of PrmA with L11 explains its observed preference for L11 as substrate before its assembly into the $50 \mathrm{~S}$ ribosomal subunit. The EMBO Journal (2007) 26, 567-577. doi:10.1038/

sj.emboj.7601508; Published online 11 January 2007

Subject Categories: structural biology

Keywords: methyltransferase; post-translational modification; X-ray crystallography

\section{Introduction}

Post-synthesis modification of proteins and nucleic acids is a ubiquitous phenomenon in biology. One of the most frequently encountered modifications is methylation, and substantial cellular effort is devoted to this process in terms of both the synthesis of donor substrate, $S$-adenosyl-L-methionine (AdoMet), and the enzymatic machinery required to catalyze the methylation reactions. AdoMet-dependent methyltransferases (MTs) form one of 15 distinct families of AdoMet binding proteins, and they are further divided into five structurally distinct classes (Schubert et al, 2003a; Kozbial and Mushegian, 2005). The catalytic domain structure of the largest class I family is characterized by a central seven-stranded $\beta$-sheet that is flanked by three helices on

\footnotetext{
${ }^{*}$ Corresponding author. Department of Molecular Biology, Cell Biology and Biochemistry, Brown University, Box G-E129, Providence, RI 2912, USA. Tel.: +1 401863 6123; Fax: + 1401863 6114;

E-mail: gerwald_jogl@brown.edu
}

Received: 26 September 2006; accepted: 27 November 2006; published online: 11 January 2007 each side and is structurally similar to Rossmann-fold domains. This catalytic domain structure is generally well conserved between family members, and substrate specificity is achieved by additional substrate recognition domains that are inserted in various regions throughout the catalytic fold (Martin and McMillan, 2002). Class I MTs recognize a wide variety of substrates, including nucleic acids and protein side chains. Among the substrates for methylation are components of the protein synthesis apparatus, including tRNAs and rRNAs (Limbach et al, 1994), ribosomal proteins L3 and L11 (Colson et al, 1979), elongation factor Tu (EF-Tu) (Vannoort et al, 1986) and polypeptide release factors 1 and 2 (RF1 and RF2) (Heurgue-Hamard et al, 2002).

The most heavily methylated component of the bacterial translational apparatus is ribosomal protein L11, a universally conserved component of the large ribosomal subunit and an active participant in interactions of the ribosome with protein synthesis factors during the initiation, elongation and termination phases of translation. L11 consists of a $23 \mathrm{~S}$ rRNAbinding C-terminal domain and an $\mathrm{N}$-terminal domain responsible for direct ribosome-factor contacts (Wimberly et al, 1999; Agrawal et al, 2001). Cryo-electron microscopic (cryoEM) reconstructions of 705 ribosome-elongation factor complexes demonstrate direct contact between the N-terminal domain of L11 and elongation factors G (EF-G) (Agrawal et al, 2001) or Tu (Stark et al, 2002; Valle et al, 2003). The formation of an arc-like connection between L11 and EF-G requires a movement of the $\mathrm{N}$-terminal domain that is facilitated by the flexible interdomain linker (Agrawal et al, 2001). This domain also comprises part of the binding site of the antibiotic thiostrepton, known primarily as an inhibitor of productive EF-G binding to the ribosome (Cameron et al, $2002,2004 \mathrm{~b}$ ), and a number of single amino-acid substitutions in the N-terminal domain confer thiostrepton resistance (Cameron et al, 2004b). The methyltransferase responsible for methylation of L11 is PrmA, encoded by the prmA gene, which trimethylates multiple residues in the L11 N-terminal domain (Vanet et al, 1993, 1994; Cameron et al, 2004a). The location of the modified residues near the site of contact with elongation factors suggests a functional role for these methylations (Cameron et al, 2004a). However, although the phylogenetic conservation of the recognition sequence and the critical functional role of the N-terminal domain of L11 have been well established, no function of the methylations by PrmA has been identified. Similar to a number of other ribosome and tRNA modifying enzymes, PrmA is dispensable, as demonstrated by the viability of prmA-null mutants of Escherichia coli (Vanet et al, 1993) and Thermus thermophilus (Cameron et al, 2004a).

PrmA in E. coli introduces a total of nine methyl groups at three amino-acid positions, trimethylating the $\alpha$-amino group of the N-terminal amino acid and the $\varepsilon$-amino group of two lysine residues, Lys3 and Lys39 (Dognin and WittmannLiebold, 1980). Matrix-assisted laser desorption ionizationtime of flight mass spectrometric analysis indicates that 
T. thermophilus PrmA trimethylates an additional fourth residue (Cameron et al, 2004a). The trimethylation of multiple, spatially dispersed residues on the same protein suggests the existence of a substrate recognition mechanism with unusual structural plasticity. However, there are currently no data clearly indicating how PrmA interacts with L11, although what is known suggests a number of testable, alternative hypotheses. PrmA may modify all four sites in a single binding event or it may dissociate and reassociate with each modification. If PrmA does adopt distinct binding modes for each modification, it may or may not have a preferred order of methylation.

A crystal structure of the apo-form of $T$. thermophilus PrmA (PDB code 1UFK; Kaminishi et al, 2003) shows PrmA to be comprised of two domains connected by an extended and potentially flexible linker, suggesting that relative domain movement may contribute to the ability of PrmA to modify multiple residues. The structure of full-length L11 from Thermotoga maritima has previously been determined in the apo-form (Ilin et al, 2005) and in the RNA-bound form (Wimberly et al, 1999). In these two structures, the C-terminal RNA-binding domain and N-terminal domain assume different orientations relative to one another. Furthermore, results from a recent NMR analysis of the L11 conformation indicate that significant domain reorientation must occur upon binding 23S rRNA via an induced fit mechanism (Ilin et al, 2005). This ability of L11 to engage in relative domain movement, and the possibility for PrmA to do so as well, suggests that domain movement of both proteins may contribute to the methylation mechanism. Finally, recombinant PrmA is catalytically active and preferentially modifies free L11 before its assembly into the 50S ribosomal subunit (Cameron et al, 2004a), but whether this is due to conformational differences between free and ribosome-bound L11, or if PrmA recognizes a portion of L11 that is unavailable in the intact $50 \mathrm{~S}$ subunit has not previously been determined.

With the ultimate goal of dissecting the PrmA-L11 recognition mechanism, we determined the structure of PrmA from T. thermophilus HB8 in four distinct conformations: two apoenzyme structures, a PrmA-AdoMet enzyme-cofactor complex and a PrmA-L11 enzyme-substrate complex. Our results reveal how PrmA binds to L11 and show clearly that a wide range of domain movements of PrmA are possible, which in turn suggests how a single enzyme can trimethylate residues at multiple positions on L11 without necessitating dissociation of the enzyme-substrate complex.

\section{Results}

\section{The structure of PrmA}

We determined the structure of PrmA in three different space groups and in a complex with its substrate, ribosomal protein L11. The structure of PrmA in space group C2 (hereafter referred to as PrmA1) was solved by molecular replacement with PDB entry 1UFK (Kaminishi et al, 2003) as a search model to $1.59 \AA$ resolution. We subsequently crystallized PrmA in two other space groups, $\mathrm{P} 22_{1} 22$ (PrmA2, $2.3 \AA$ resolution) and R3 (PrmA3 in complex with the cofactor AdoMet, $1.75 \AA$ resolution). Crystals in space groups $\mathrm{P} 22_{1} 2_{1} 2$ and R3 were obtained from crystallization experiments for the protein complex between PrmA and L11 containing a
K39A single-site mutation. PrmA forms a tight-binding complex with the L11 K39A mutant protein. These experiments aimed to trap a second complex conformation; however, instead of the protein-protein complex, we obtained crystals of PrmA. Despite continued efforts, we have not been able to obtain complex crystals with L11 K39A. For structure solution by molecular replacement in space groups $\mathrm{P}_{1} 2_{1} 2$ and R3, the PrmA N-terminal domain (residues 1-54) and C-terminal domain (residues 69-254) had to be located separately and the connecting region between the domains was built manually. For all three data sets, the majority of residues (95.5, 89.9 and $93.7 \%$ ) are in the most favored region of the Ramachandran plot with no residues in the disallowed region.

In PrmA1 and PrmA2, all residues are well defined in the electron density map with the exception of residues 97-101, which are disordered and were omitted from the final model. Clear electron density for residues 97-101 was however found in the presence of AdoMet in data set PrmA3. This region is in close proximity to the AdoMet-binding site, and defines a highly conserved sequence motif in all PrmAs (Figure 1). Two glycine residues (Gly96 and Gly102) form flexible hinges on both sides of a short loop (hereafter referred to as the glycinerich loop) that closes over the bound cofactor (discussed below).

PrmA consists of two domains that are connected by a flexible linker helix (Figure 2A and B). The large C-terminal catalytic domain forms a canonical class I Rossmann-like methyltransferase fold with high structural similarity to PrmC. A central seven-stranded $\beta$-sheet is flanked by helices on both sides. A small additional three-stranded $\beta$-sheet is tightly anchored to the Rossmann-like domain and serves as an anchor for the linker helix $\alpha 2$. The small N-terminal domain consists of a four-stranded $\beta$-sheet that is partially flanked by the linker helix $\alpha 2$ and the N-terminal helix $\alpha 1$ on each side. The N-terminal domain is unique to PrmA and is capable of binding to the substrate protein L11 on its own (data not shown). Intriguingly, a database search with the program DALI (Holm and Sander, 1993) revealed that the PrmA N-terminal domain (residues 1-54) is structurally related to domain $\mathrm{V}$ of the ribosomal translation elongation factor G (EF-G, Dali Z-score 5.3, $53 \mathrm{C} \alpha$ atoms aligned with $2.4 \AA$ r.m.s.d.), which is located in close proximity to protein L11 in cryo-EM reconstructions of ribosomes complexed with EF-G (Agrawal et al, 2001).

Least-squares alignment of the PrmA catalytic domains of the three different crystal forms and the protein complex structure illustrates a remarkable conformational flexibility of the $\mathrm{N}$-terminal domain with respect to the catalytic domain (Figure 2C). The N-terminal and C-terminal domains remain basically unchanged in all four crystal structures (r.m.s.d.s of $0.61 \AA$ between PrmA1 and PrmA2, $0.354 \AA$ between PrmA1 and PrmA3 and $0.81 \AA$ between PrmA1 and the complex structure for residues 1-54; r.m.s.d.s of $0.48,0.49$ and $0.44 \AA$, respectively, for the catalytic domain between residues 70 and 254). However, the domain location with respect to the catalytic domain is unique in each crystal form. Helix $\alpha 2$ serves as a rotation axis for a rigid-body rotation of the $\mathrm{N}$-terminal domain of approximately $55^{\circ}$ between PrmA1 and PrmA2 and approximately $180^{\circ}$ between PrmA1 and the complex structure. For the cofactor-bound form PrmA3, we observed an additional swiveling domain motion that tilts the 
A

Thermus/1-254 Escherichia/1-293

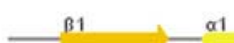

$\alpha 1$ $\beta 2$ $\beta 3$ $\beta 4$ 1. MWVYRLKGTLEALDPILPGLFDGGARGLWERE ... GEVWAFFP. . . . . . . . . APVDLPYEGVWEE 53 1 MPWIQLKLNTTGANAEDLSDALMEAGAVSITFQDTHDTPVFEPLPGETRLWGDTDVIGLFDAETDIMNDVVAILEN 75

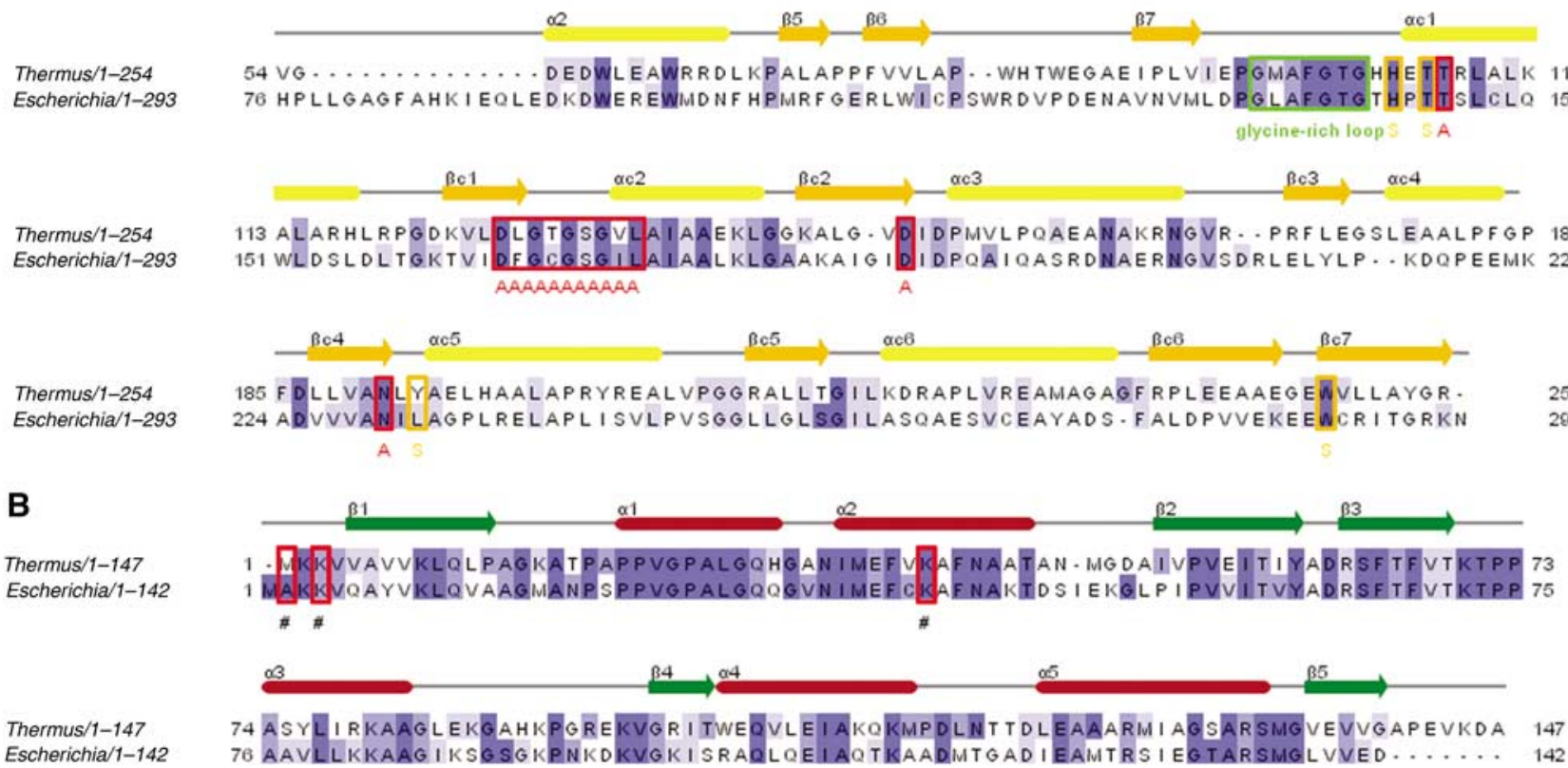

Figure 1 Structure-based sequence alignment of PrmA and ribosomal protein L11. (A) Structure-based sequence alignment of PrmA. Color coding represents the percentage identity for a sequence alignment of PrmA homologs from nine different bacterial phyla (see Supplementary Figure S1 for full alignment). Secondary structure elements are indicated on top based on the PrmA3 structure. The position of the flexible glycine-rich loop is marked with a green box. Residues in contact with AdoMet are enclosed in red boxes and labeled 'A', and residues in the active site are indicated with orange boxes and labeled 'S'. (B) Sequence alignment of protein L11. Color coding represents the percentage identity for a sequence alignment from nine different phyla (see Supplementary Figure S2 for full alignment). Secondary structure elements are indicated on top based on the L11 structure in complex with RNA (PDB code 1MMS). The position of substrate lysine residues for E. coli L11 are labeled ' \#'.

$\mathrm{N}$-terminal domain by about $45^{\circ}$ and positions it closer to the active site than in all other structures. The large rotation between the different crystal forms suggests that the location of the PrmA N-terminal domain with respect to the catalytic domain is not stabilized by interdomain contacts and probably moves freely in solution. Overall, the observed high flexibility of the N-terminal substrate recognition domain is consistent with the unique requirement of PrmA to position its protein substrate in several different orientations in order to methylate several side chains, and suggests that PrmA is capable of fully methylating all side chains without releasing the substrate.

\section{The cofactor-binding site}

Crystal forms PrmA1 and PrmA2 consist of the apo-enzyme. We were unable to obtain cofactor-bound forms of these crystals by either soaking or co-crystallizing with AdoMet or S-adenosyl-L-homocysteine (AdoHcy). The cofactor-bound crystal form PrmA3 was obtained during attempts to cocrystallize the PrmA-L11 K39A complex in the presence of AdoMet. These crystals were found to contain PrmA alone with AdoMet in the cofactor-binding site. Cofactor electron density is well defined in the $1.75 \AA$ electron density map of PrmA3 (Figure 3B).

As seen in other class I methyltransferases, AdoMet is bound in a canonical conformation above the $\beta$-sheet and close to the conserved GxGxG signature motif between strand $\beta \mathrm{c} 1$ and helix $\alpha \mathrm{c} 2$ (Figure 3A). The adenine ring is located in a hydrophobic pocket that is lined by residues Leu127, Ile150, Leu192 and Leu196. The N1 atom of adenine is recognized specifically by the methyltransferase through a hydrogen bond to Ser175, which anchors the adenosine in the hydrophobic pocket, whereas the adenine N6 and N7 atoms form additional favorable interactions with solvent water molecules W164 and W226. Both ribose hydroxyl groups form hydrogen bonds with the strictly conserved Asp149. The strictly conserved Thr107 and Asn191 recognize the AdoMet methionine carboxyls, and the amino group forms a hydrogen bond to the Gly128 (part of the GxGxG signature motif) main-chain carbonyl and two solvent water molecules. The hydrophobic Phe99 from the glycine-rich loop stacks very close to the AdoMet sulfur atom and might contribute to the enzymatic function by destabilizing the cofactor. Not surprisingly, all residues in the AdoMet-binding site are strictly conserved between all PrmAs despite lower overall sequence similarity, as is the glycine-rich loop with the central Phe99.

\section{The PrmA-L11 complex structure}

PrmA-L11 complexes readily produced large crystals $(0.22 \times 0.22 \times 1.0 \mathrm{~mm})$. However, the diffraction limit of most crystals was only between 2.5 and $3 \AA$, indicating that parts of the complex are not fully ordered in the crystal lattice. We solved the structure by molecular replacement in space group $\mathrm{P}_{5}$ using the coordinates of PrmA1 and the published coordinates of T. maritima L11 (PDB code 1MMS; 
A
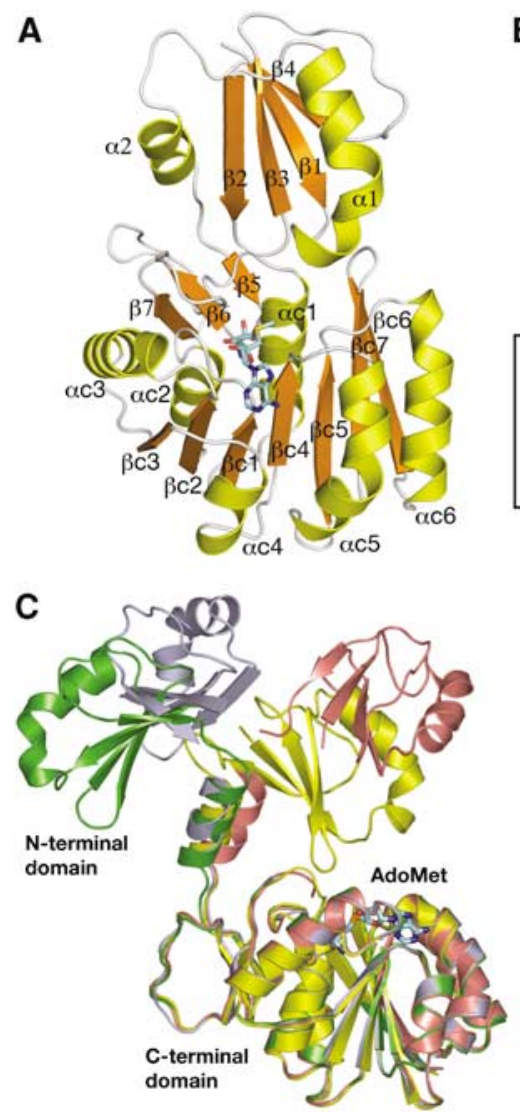

B
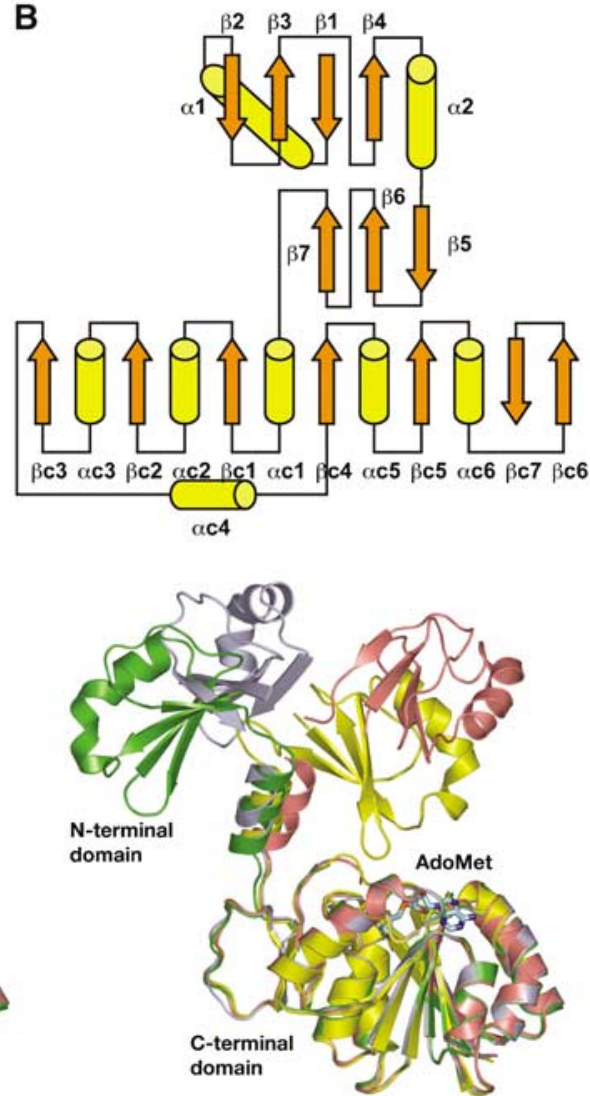

Figure 2 Structure of PrmA. (A) Cartoon representation of the cofactor-bound form of PrmA (PrmA3). AdoMet is shown in a stick representation with carbon atoms colored in cyan. (B) Topology diagram of PrmA. (C) Stereo representation of four different conformations of PrmA. Apo-enzyme structures of PrmA1 and PrmA2 are colored in green and blue, respectively. The AdoMet-bound form PrmA3 is colored in yellow. The structure of PrmA in the complex is colored in salmon.

Wimberly et al, 1999) as a search model. The PrmA C-terminal catalytic domain and the L11 N-terminal domain could be located automatically, but all available molecular replacement packages failed to locate the other two domains. To place the L11 C-terminal domain and the PrmA N-terminal domain, we partially traced both domains in difference electron density maps obtained from the partial solution and placed the missing domains by least-squares alignment with the manually built model. In the final model, the electron density for the PrmA catalytic domain and the L11 $\mathrm{N}$-terminal domain is well defined (Figure 4B), but the electron density for the PrmA N-terminal domain and the L11 C-terminal domain is weaker, indicating that both domains are partially disordered in the complex crystal. The average temperature factors for the PrmA catalytic domain and the L11 N-terminal substrate domain are 38 and $62 \AA^{2}$, respectively, compared with 94 and $97 \AA^{2}$ for the PrmA $\mathrm{N}$-terminal domain and the L11 C-terminal domain (restricting $B$ factors to a maximum value of 100 during refinement).

In the final model for the complex structure, the PrmA $\mathrm{N}$-terminal domain assumes yet another orientation as compared with the three PrmA-only structures (Figures $2 \mathrm{C}$ and $4 \mathrm{~A})$. The conformations of both the catalytic and the $\mathrm{N}$-terminal domains are similar to those observed in the unbound PrmA structures with an r.m.s.d. of $0.44 \AA$ for the catalytic domain and $0.81 \AA$ for the $\mathrm{N}$-terminal domain relative to PrmA1. In contrast to previous structures of protein L11, the first seven residues are ordered in our complex structure, mainly owing to a crystal contact between the L11 N-terminal amino group and a Glu85 carboxylate from a neighboring PrmA molecule.

As anticipated, the N-terminal substrate recognition domain forms extensive contacts with L11. Complex formation buries a surface area of $1677 \AA^{2}$ of the total PrmA surface of $11409 \AA^{2}$, with a contribution of $1228 \AA^{2}$ from the PrmA $\mathrm{N}$-terminal domain and linker region (residues 1-68). Despite the large complex interface, only seven hydrogen bonds are formed between the proteins and only two of these involve the PrmA catalytic domain. The PrmA N-terminal domain recognizes L11 with three main motifs, two of which contact the L11 N-terminal domain (Figure 4C). An extended $\beta$-sheet is formed connecting the N-terminal L11 $\beta$-sheet with $\beta 4$ from the PrmA N-terminal domain. The second motif consists of a hydrophobic interface formed by Trp29, Phe38 and Trp59 (from the PrmA linker helix $\alpha 2$ ) that interacts with the highly conserved Pro-rich loop region of L11. Finally, helix $\alpha 1$ inserts between both L11 domains (Figure 4D). In this third motif, Phe21 of helix $\alpha 1$ forms hydrophobic interactions with Pro13 and Ile52 from the L11 N-terminal domain, and L11 Ser75 and Lys86 establish hydrogen bonds with main-chain carbonyls from Pro15 and Gly19 of PrmA. In our model, PrmA Asp22 and L11 Arg79 are positioned to form a salt bridge between both proteins, but remain at a distance of $3.8 \AA$. It is however conceivable that a reorientation of both proteins establishes this interaction during methylation of another L11 residue. 


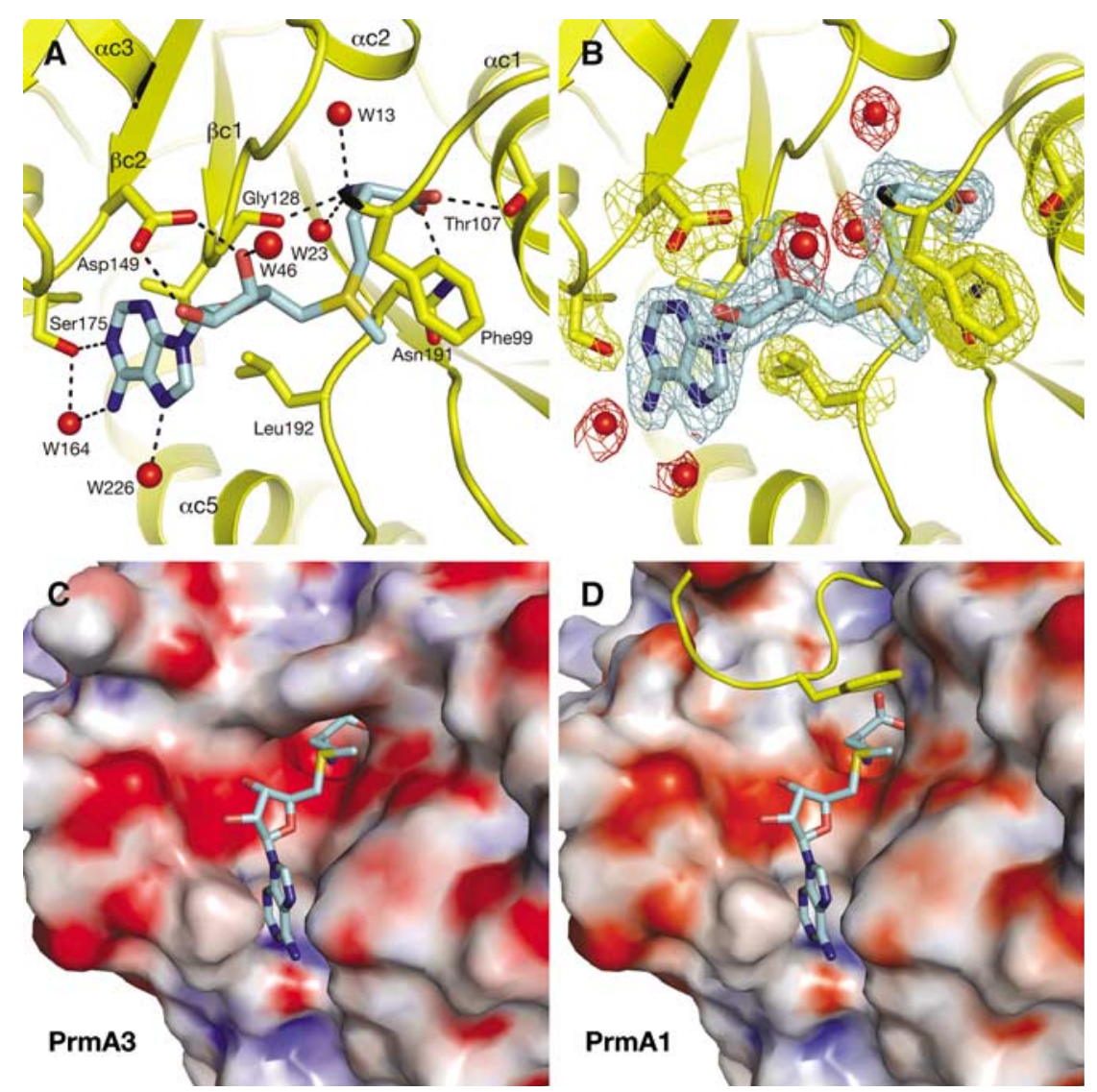

Figure 3 The AdoMet-binding site in PrmA. (A) Ribbon diagram of the AdoMet-binding site. Cofactor-interacting side chains are shown as sticks; solvent water molecules are represented with red spheres. (B) Final $2 F_{\mathrm{O}}-F_{\mathrm{c}}$ density for AdoMet, interacting PrmA side chains and solvent water molecules contoured at the $1.3 \sigma$ level. (C, D) Contribution of the glycine-rich loop to the cofactor-binding site. Surface illustration of PrmA colored by electrostatic potential in the presence (C) and in the absence (D, loop position indicated by a cartoon for reference) of the glycine-rich loop.

In contrast to the substrate recognition interface, the interface between the L11 N-terminal domain and the PrmA catalytic domain is substantially smaller with $466 \AA^{2}$ of buried surface area (Figure 4D). L11 helix $\alpha 2$ is positioned across the surface of the catalytic domain with the side chain of Lys39 projecting into the active site. The interface region is mostly dominated by hydrophobic interactions and hydrogen bonds between PrmA Arg223 and L11 Asn47 and between PrmA Asn191 and L11 Lys39. Tyr193 is the only residue in the PrmA interface area that changes its orientation and rotates toward Ala43 and Ala46 of the L11 helix $\alpha 2$.

Protein L11 is bound to PrmA in an overall conformation closely resembling the rRNA-bound form (r.m.s.d. $1.8 \AA$; Figure $4 \mathrm{E})$. One important conclusion drawn from the structure of the complex is that the binding surfaces of L11 used to interact with rRNA and PrmA overlap, precluding simultaneous binding and thereby inhibiting modification of ribosome-bound L11. This observation explains previous biochemical data indicating the preferential methylation of L11 before its incorporation into the $50 \mathrm{~S}$ ribosomal subunit (Cameron et al, 2004a).

In summary, the PrmA N-terminal domain forms the majority of contacts by a combination of shape complementarity, $\beta$-sheet formation between both proteins and recognition of key structural features of L11, such as the proline-rich loop. The smaller interface with the catalytic domain is dominated by hydrophobic interactions that establish a favor- able but nonspecific protein-protein contact. The observed binding pattern is consistent with the requirements for specific substrate recognition with retention of conformational flexibility to position different side chains in the active site.

\section{The PrmA active site}

Despite intense efforts to obtain ternary complexes, we were unable to observe electron density for AdoMet or AdoHcy in several complex structures. We therefore modeled the position of AdoMet in the PrmA-L11 complex by least-squares alignment of PrmA with the cofactor-bound structure PrmA3. Similar to the apo-PrmA structures, the glycine-rich loop is disordered in the complex structure in the absence of the cofactor. However, only residues 99 and 100 could not be traced and were omitted from the final model. A comparison of the PrmA surfaces formed in the absence and presence of cofactor shows that the loop region is not directly involved in the binding interface and the cofactor-binding site is significantly more open in the absence of the loop (Figure 5).

In the PrmA-L11 complex structure, Lys39 of L11 assumes an extended conformation that inserts into the PrmA active site (Figure 5). The bottom of the active site cavity is formed by Asn191, Thr106 and Thr107, whereas the wall is lined by Phe99, Tyr193, Trp247 and His104. The amino group of Lys39 of L11 is positioned at a distance of $2.6 \AA$ from the AdoMet methyl group and $3.3 \AA$ from Asn191, which also coordinates to the AdoMet carboxylates. There are no residues present 
A
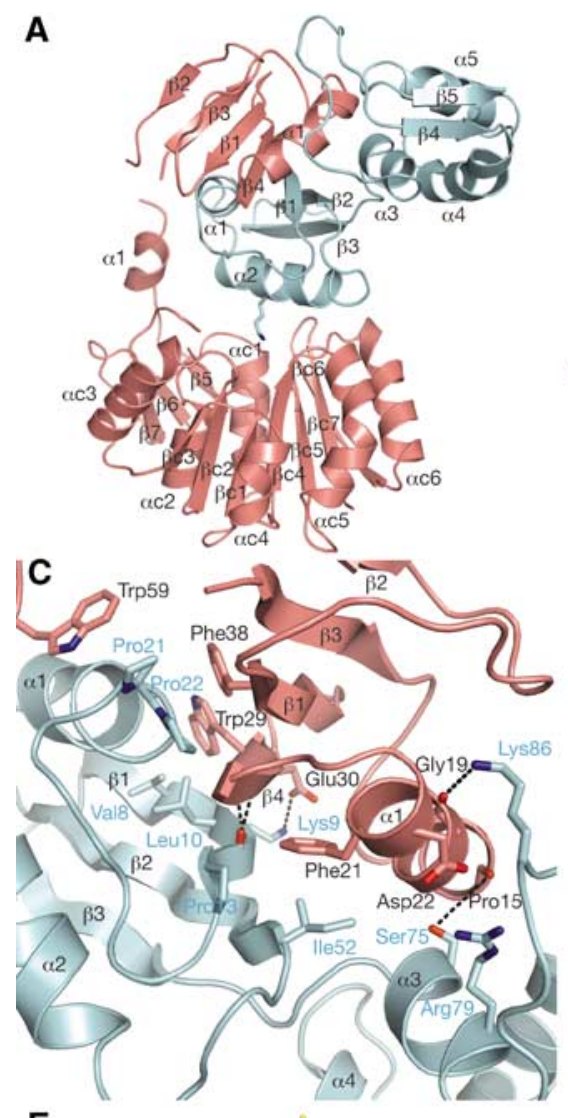

$\mathrm{E}$

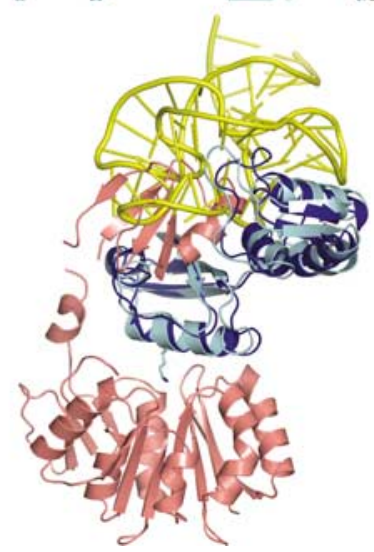

B
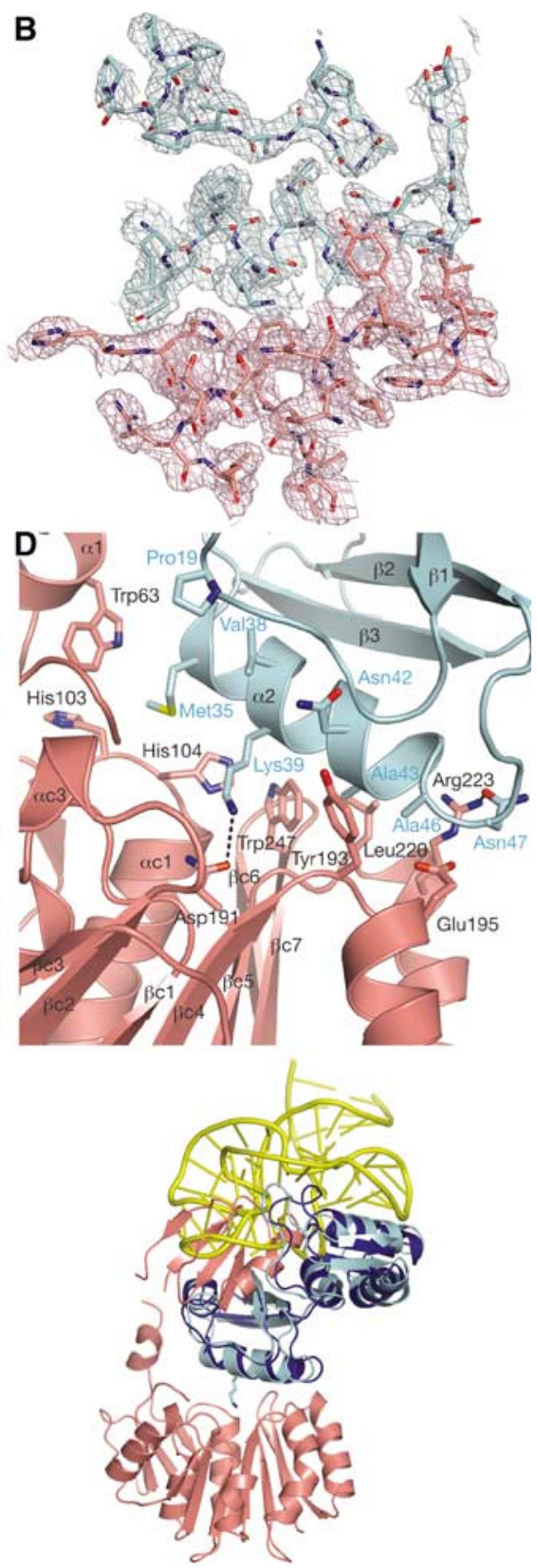

Figure 4 The PrmA-L11 complex structure. (A) Cartoon representation of the overall structure. PrmA and L11 are colored in salmon and cyan. The Lys39 side chain is shown in a stick representation. (B) Final $2 F_{\mathrm{o}}-F_{\mathrm{c}}$ density for the active site region contoured at the $1 \sigma$ level. (C) Ribbon diagram of binding interactions between the PrmA N-terminal domain and L11. (D) Ribbon diagram of binding interactions between the PrmA catalytic domain and L11. (E) Stereo representation comparing the PrmA-L11 complex with the L11-RNA complex (PDB code 1MMS) after least-squares alignment of L11. PrmA is colored in salmon and L11 is colored in cyan in the PrmA complex and in blue in the RNA complex. RNA is shown in yellow.

that could restrict the rotational movement of the Lys39 side chain, as would be expected for a trimethyltransferase.

Lysine trimethylation by a processive catalytic mechanism requires the presence of a general base for substrate deprotonation. A potential general base, His104, is placed on the opposite side of the cofactor at a distance of $5.3 \AA$ between the ND1 nitrogen and the Lys39 amino group and forms hydrogen bonds to Thr106 and a solvent water molecule, W182. It is conceivable that a rotation of the lysine side chain could bring the amino group in close proximity to His104 acting as a general base to accept a proton and later exchange it with solvent water molecules. A functional role for this residue is also supported by the observation that His104, the coordinating Thr106 and Trp247 are strictly conserved in the PrmA family (Figure 1). The only other residue that could act as a general base is Tyr193, but this residue is not conserved among the PrmAs, and the Tyr193 hydroxyl group is positioned unfavorably and further removed (7.1 $\AA$ ) from Lys39.

\section{Discussion}

Methylation of L11 by PrmA is unique in its requirement for simultaneous specific recognition and flexible reorientation during catalysis. This is reflected in the remarkable flexibility 


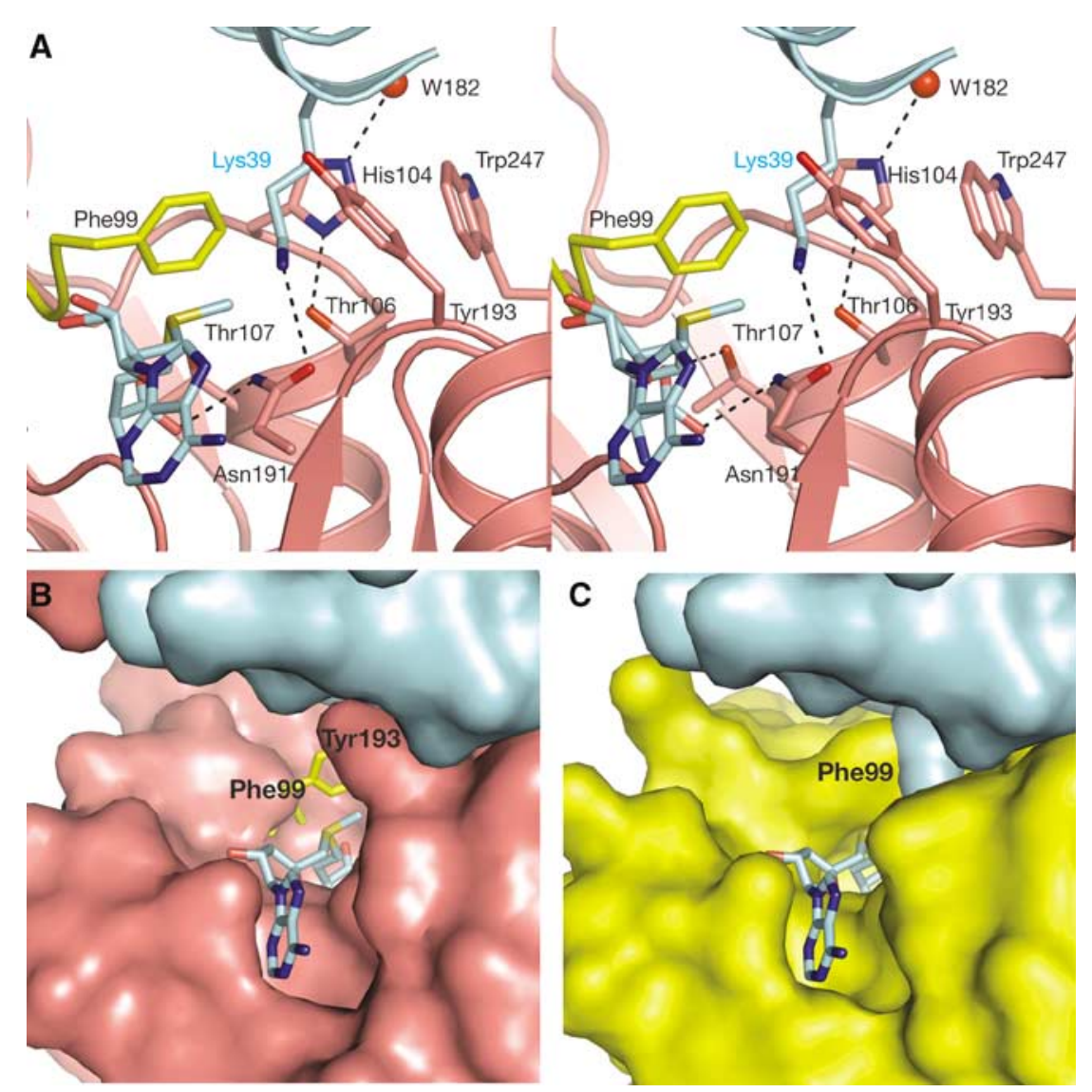

Figure 5 The active site of PrmA. (A) Stereo representation of the active site. The position of AdoMet and Phe99 is modeled by least-squares alignment from the position observed in PrmA3. (B, C) Comparison of the PrmA surface in the complex structure (B, flexible loop absent) with the cofactor-bound structure PrmA3 (C, flexible loop present, L11 surface is shown for reference).

of the PrmA N-terminal domain with respect to the catalytic domain, as demonstrated by the four different PrmA conformations reported in this study. E. coli PrmA trimethylates Lys3, Lys39 and the $\alpha$-amino group of the N-terminal amino acid (Dognin and Wittmann-Liebold, 1980), whereas T. thermophilus PrmA is expected to methylate these same positions and an additional fourth side chain. Clearly, a very different orientation of L11 with respect to PrmA is required to place the different substrate side chains into the active site. Modeling L11 in a complex with PrmA in its PrmA1 or PrmA2 conformation positions Lys 3 in the same plane with the active site such that a simple rotation around the PrmA linker helix $\alpha 2$ will suffice to move Lys3 close to the active site entrance (Supplementary Figure S3).

Despite the unique overall domain structure of PrmA, the catalytic domain of this enzyme is similar to that of other methyltransferases. This is particularly true of PrmC (Graille et al, 2005), wherein a $\beta$-hairpin and a linker helix are located in a position similar to that of the three-stranded anchoring $\beta$-sheet and linker helix of PrmA (Figure 6A). Further, the PrmC-RF1 complex structure shows that the positions of the cofactor and the substrate side chain (Lys39 and Gln235) are similar in both methyltransferases (Figure 6B). The structural similarity even extends to the position of the PrmA glycinerich loop, where Leu89 of E. coli PrmC (or Phe100 in T. maritima; Schubert et al, 2003b) assumes a very similar position to the central PrmA Phe99. However, the observed structural flexibility seems to be unique to PrmA, as the glycine loop hinges are not present in PrmC and there is no indication that the loop region of PrmC becomes disordered in the absence of substrate. In contrast to PrmC, the trimethyltransferase PrmA needs to exchange the cofactor twice if lysine trimethylation occurs by a processive mechanism. The strict conservation of the glycine-rich loop sequence and the retention of the cofactor-binding geometry suggest that the observed loop flexibility establishes an elegant mechanism to facilitate cofactor access to the active site and that loop closure might even initiate the enzymatic reaction via the close contact of Phe99 with the AdoMet sulfur atom. This observation therefore suggests a processive catalytic mechanism for lysine trimethylation.

While the PrmA-L11 complex structure is the first substrate-complex structure for a class I protein trimethyltransferase, detailed studies have been performed regarding the structural determinants for mono- and trimethylation reactions in the structurally unrelated histone lysine methyltransferases (Trievel, 2004). In the SET7/9 monomethyltransferase (Xiao et al, 2003), a tyrosine residue and a solvent water molecule form hydrogen bonds with the substrate lysine amine, effectively restricting the rotational movement of the amino group after the first methylation reaction. Removal of the tyrosine side chain by mutagenesis broadens the enzymatic specificity from a monomethyltransferase to a trimethyltransferase. The opposite conversion of the trimethyltransferase DIM-5 to a mono- and dimethyltransferase is achieved by introducing an additional tyrosine residue in the active site (Zhang et al, 2003). Despite the unrelated architecture of the PrmA active site, lysine binding follows 
A

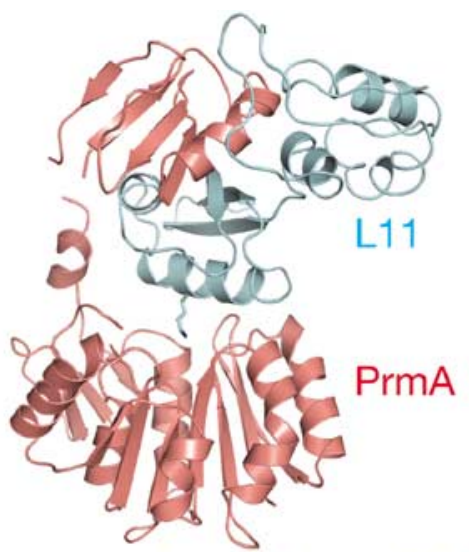

B
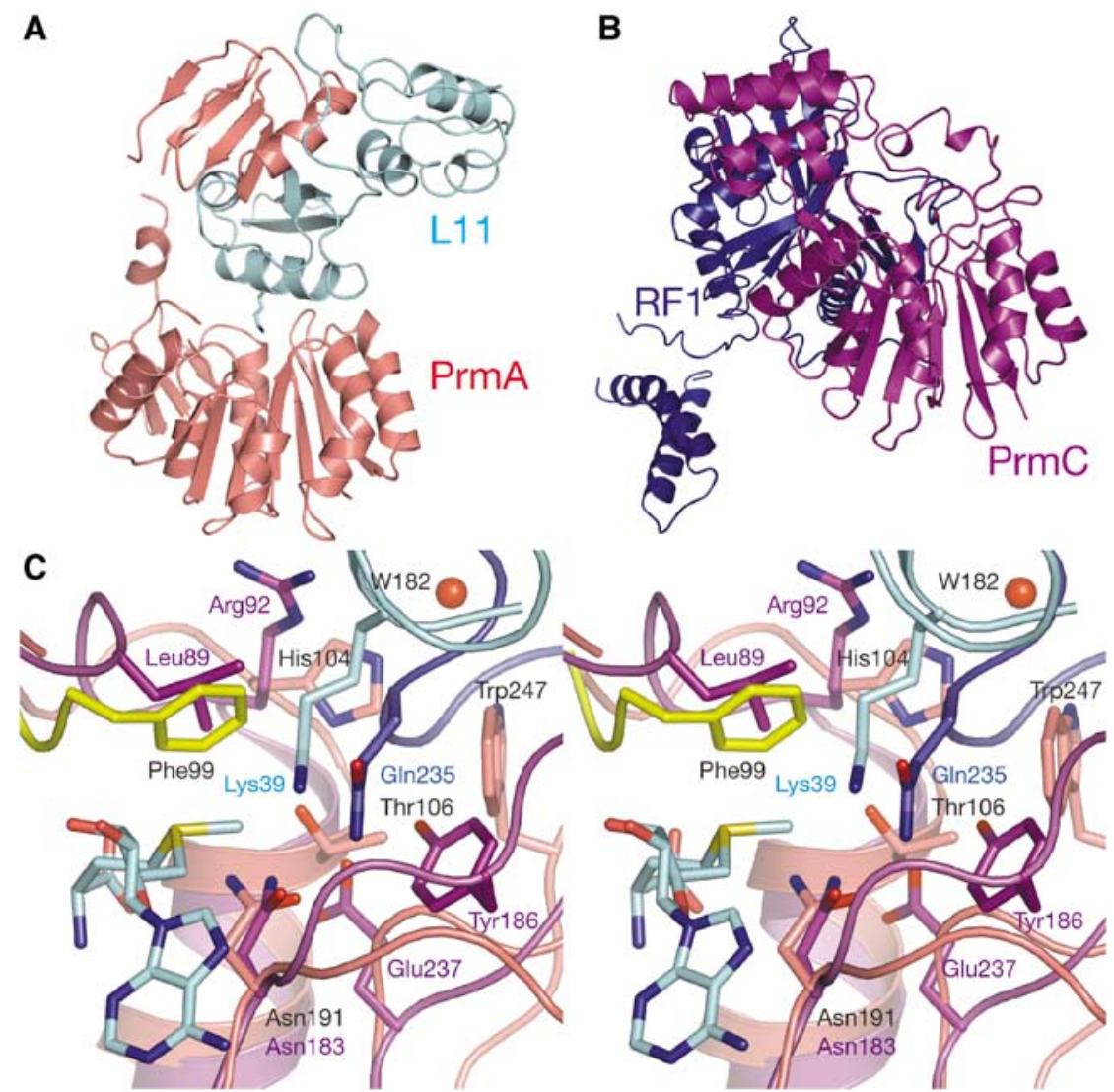

Figure 6 Comparison of the substrate complexes of PrmA and PrmC. (A, B) Comparison of the PrmA-L11 complex with the PrmC-RF1 complex (PDB code 2B3T). PrmA-L11 is colored as before, PrmC is colored in purple and RF1 is colored in blue. (C) Stereo representation of the active site region illustrating the similarity of the position of substrate side chains Lys39 (PrmA) and Gln235 (PrmC), the flexible loop region and the cofactor coordinating Asn191 (PrmA)/Asn183 (PrmC). Color scheme as in (A) and (B); the position of AdoMet and Phe99 (colored in yellow) is modeled based on the PrmA3 structure.

similar principles as observed in the SET domain trimethyltransferases. First, the substrate lysine inserts into the active site through a tunnel-like region lined with hydrophobic residues that interact with the alkyl portion. Next, however, instead of coordinating with tyrosines, the amino group is oriented toward AdoMet by a hydrogen bond to Asn191, and finally, there are no additional interacting residues present to restrict rotation of the amino group after the first methylation reaction.

There are currently no data giving a clear indication of the role of L11 methylation. The overlapping of L11-binding sites for PrmA and rRNA as observed in our complex structure makes a role for PrmA as an assembly factor somewhat unlikely, and the location of methylation sites distant from the RNA-binding surface does not support a direct role for the methylations themselves in the assembly process. It is tempting to speculate that methylation might influence the interaction of L11 with any of a number of protein factors. L11 Lys39 on the surface of helix $\alpha 2$ is oriented toward the ribosome surface in a position that is unlikely to interact with either elongation or release factors, whereas Lys3 is positioned such that methylation of this residue could potentially influence interaction with EF-G. L11 methylation does not influence the stringent response (Rohl and Nierhaus, 1979), although there are other GTPases that interact with the ribosome, and probably with L11 directly, including members of the Obg family of GTPases, which participate in a variety of cellular response pathways (Kukimoto-Niino et al, 2004).

The dispensibility of PrmA excludes by no means a direct role for methylation of L11 in normal ribosome function. This point is perhaps most clearly illustrated by observations with PrmC. PrmC methylates the N5 position of Q252 in the conserved GGQ motif of RF1 and RF2 (Heurgue-Hamard et al, 2002), and this methylation has been shown to enhance polypeptide release activity in vitro (Dincbas-Renqvist et al, 2000). Deletion of prmC in E. coli results in a severe growth defect, but only in the context of a strain-specific mutation of the highly conserved Ala246 of RF2; the growth defect of the prmC-null allele is cured by 'reversion' of Thr246 to Ala. By analogy, the contribution of L11 methylation may be to modulate the accuracy or kinetics of some aspect of ribosome function, such as decoding or translocation, but the contribution of methylation may be detectable only by careful kinetic analyses in vitro. The contribution of methylation may manifest itself in vivo only in concert with specific mutations in L11 or in some other component of the protein synthetic apparatus.

\section{Materials and methods}

Construction of an E. coli strain for overexpression of unmethylated $T$. thermophilus ribosomal protein $L 11$

E. coli BL21 (DE3) was transduced to prmA::Tn10 using P1vir phage grown on KNOK16 (prmA::Tn10) (Vanet et al, 1994) according to 
standard protocols (Miller, 1992). Tetracycline-resistant transductants were purified by restreaking twice for single colonies to eliminate P1 lysogens. The resultant strain was designated HD1.

\section{Protein expression, purification and crystallization}

Full-length PrmA from T. thermophilus HB8 was subcloned into the pET30b vector (Novagen) and overexpressed in E. coli strain BL21DE3 (Invitrogen) at $293 \mathrm{~K}$. Bacterial cells were lysed by ultrasonification on ice in a buffer containing $20 \mathrm{mM}$ Tris $(\mathrm{pH}$ 8.5), $300 \mathrm{mM} \mathrm{NaCl}, 5 \mathrm{mM} \beta$-mercaptoethanol, $0.1 \%$ Triton-X 100 and $5 \%$ glycerol. The soluble protein was incubated at $338 \mathrm{~K}$ for $30 \mathrm{~min}$. Precipitated E. coli proteins were separated by centrifugation and the soluble PrmA was further purified with anion exchange chromatography using a linear gradient of $10 \mathrm{mM}$ to $1 \mathrm{M} \mathrm{NaCl}$ concentration and size-exclusion chromatography at $\mathrm{pH} 8.5$ and $200 \mathrm{mM} \mathrm{NaCl}$ and $1 \mathrm{mM}$ DTT. Purified PrmA was concentrated to $24 \mathrm{mg} / \mathrm{ml}$ in a buffer containing $20 \mathrm{mM}$ Tris (pH 8.0), $150 \mathrm{mM} \mathrm{NaCl}$ and $1 \mathrm{mM}$ DTT for crystallization trials. Full-length T. thermophilus L11 was subcloned into the pET11a vector (Novagen) and overexpressed in a BL21 derivative bearing a prmA::Tn10-null allele to obtain the unmethylated form. Soluble L11 was purified in a manner similar to PrmA with the exception that cation exchange chromatography was used. For the formation of the PrmA-L11 complex, purified proteins were mixed in $20 \mathrm{ml}$ of $20 \mathrm{mM}$ Tris (pH 8.0 ) and incubated at $277 \mathrm{~K}$ for $2 \mathrm{~h}$. The complex was then gradually concentrated by centrifugation and further purified by sizeexclusion chromatography. The purified complex was concentrated to $16 \mathrm{mg} / \mathrm{ml}$ in a buffer containing $20 \mathrm{mM}$ Tris (pH 8.0).

Crystals of PrmA in space group C2 were obtained with the sitting drop vapor diffusion method at $293 \mathrm{~K}$. The reservoir solution

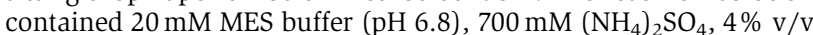
dioxane and $220 \mathrm{mM}$ hexanediol. Initial crystals grew over the course of 2-3 weeks. Streak seeding into pre-equilibrated solution produced large crystals after 1 month with maximum dimensions of $0.5 \times 0.15 \times 0.15 \mathrm{~mm}$.
Crystals in space group P21212 were obtained by sitting drop vapor diffusion at $293 \mathrm{~K}$ of the PrmA-L11 K39A complex against a well solution of $85 \mathrm{mM}$ HEPES buffer ( $\mathrm{pH} 7.5$ ) containing $1.7 \mathrm{M}$ $\left(\mathrm{NH}_{4}\right)_{2} \mathrm{SO}_{4}, 1.7 \% \mathrm{w} / \mathrm{v}$ PEG400 and $15 \% \mathrm{v} / \mathrm{v}$ glycerol. Crystals in space group R3 were grown with the microbatch method using Al's oil (Hampton Research). A $1 \mu \mathrm{l}$ portion of protein solution was mixed with $1 \mu \mathrm{l}$ of a solution containing $95 \mathrm{mM}$ HEPES $(\mathrm{pH}$ 7.5), $190 \mathrm{mM} \mathrm{CaCl}_{2} \cdot 2 \mathrm{H}_{2} \mathrm{O}, 26.6 \% \mathrm{v} / \mathrm{v}$ PEG400, and $5 \% \mathrm{v} / \mathrm{v}$ glycerol. Crystals were grown overnight to a maximum size of $0.2 \times 0.16 \times 0.16 \mathrm{~mm}$ in space group $\mathrm{P}_{1} 2_{1} 2$ and $0.44 \times 0.22 \times$ $0.22 \mathrm{~mm}$ in R3. The PrmA-L11 complex was crystallized by sitting drop vapor diffusion at $293 \mathrm{~K}$ against a reservoir solution containing $85 \mathrm{mM}$ Tris buffer (pH 8.5), 25.5\% w/v PEG4000, $120 \mathrm{mM} \mathrm{NaAc}$ $15 \% \mathrm{v} / \mathrm{v}$ glycerol and $4 \% \mathrm{v} / \mathrm{v}$ 1,1,1,3,3,3-hexafluoro-2-propanol. Complex crystals were grown overnight to a maximum size of $1.0 \times 0.22 \times 0.22 \mathrm{~mm}$.

\section{Data collection, structure determination and refinement}

Crystals were flash-frozen in liquid nitrogen directly from their mother liquor, except for monoclinic PrmA, which was cryoprotected with the addition of $50 \%$ v/v 1,2-propanediol. Diffraction data for PrmA were collected on an ADSC CCD detector at the X4A beamline of the National Synchrotron Light Source in Brookhaven at a wavelength of $0.979 \AA$ and $100 \mathrm{~K}$. Diffraction data for the complex were collected on a Mar345 detector at the X4C beamline at a wavelength of $0.979 \AA$ at $100 \mathrm{~K}$. A single crystal was used for each data set. The diffraction images were processed and scaled with the HKL2000 package (Otwinowski and Minor, 1997). The data processing statistics are summarized in Table I.

The coordinates of data set PrmA1 and T. maritima L11 (PDB 1MMS; Wimberly et al, 1999) were used as initial search models for structure determination by molecular replacement. The $\mathrm{N}$ - and C-terminal domains of PrmA were located independently with the programs COMO (Jogl et al, 2001) and Phaser (McCoy et al, 2005). The final model contains residues 1-95 and 102-254 for PrmA in

Table I Data collection and refinement statistics

\begin{tabular}{|c|c|c|c|c|}
\hline & PrmA1 & $\operatorname{PrmA2}$ & $\operatorname{PrmA3}$ & Complex \\
\hline \multicolumn{5}{|l|}{ Data collection } \\
\hline Space group & $\mathrm{C} 2$ & $\mathrm{P} 2{ }_{1} 2_{1} 2$ & $\mathrm{R} 3$ & $\mathrm{P}_{5}$ \\
\hline \multicolumn{5}{|l|}{ Cell dimensions } \\
\hline$a, b, c(\AA)$ & $81.5,76.6,61.9$ & $124.2,153.6,33.3$ & $187.2,187.2,45.2$ & $132.8,132.8,46.0$ \\
\hline$\alpha, \beta, \gamma(\mathrm{deg})$ & $90,130.01,90$ & $90,90,90$ & $90,90,120$ & $90,90,120$ \\
\hline Resolution $(\AA)^{\mathrm{a}}$ & $30-1.59(1.65-1.59)$ & $30-2.3(2.38-2.3)$ & $30-1.75(1.81-1.75)$ & $30-2.4(2.49-2.40)$ \\
\hline$R_{\text {merge }}^{\mathrm{b}}$ & $0.057(0.385)$ & $0.082(0.417)$ & $0.046(0.43)$ & $0.077(0.395)$ \\
\hline$I / \sigma I$ & $17.96(2.6)$ & $15.4(2.2)$ & $19.13(2.1)$ & $17.6(2.8)$ \\
\hline Completeness (\%) & $96.2(91.2)$ & $96.8(85.8)$ & $99.6(99.5)$ & $98.9(96.9)$ \\
\hline Redundancy & $3(2.7)$ & $3.7(2.9)$ & $2.5(2.2)$ & $4.3(3.3)$ \\
\hline \multicolumn{5}{|l|}{ Refinement } \\
\hline Resolution $(\AA)$ & $1.59(1.63-1.59)$ & $2.3(2.36-2.30)$ & $1.75(1.79-1.75)$ & $2.4(2.46-2.4)$ \\
\hline No. of reflections & $35627(2422)$ & $27095(1742)$ & $56389(4133)$ & 17389 (1229) \\
\hline$R_{\text {work }} / R_{\text {free }}^{\mathrm{c}}$ & $0.19 / 0.22(0.25 / 0.31)$ & $0.21 / 0.28(0.27 / 0.34)$ & $0.20 / 0.22(0.28 / 0.33)$ & $0.22 / 0.27(0.26 / 0.39)$ \\
\hline \multicolumn{5}{|l|}{ No. of atoms } \\
\hline Protein & 1923 & 3799 & 3860 & 2959 \\
\hline Ligand/ion & - & - & 54 & - \\
\hline Water & 168 & 292 & 296 & 92 \\
\hline \multicolumn{5}{|l|}{$B$-factors } \\
\hline Protein & 26 & 48 & 31 & 63 \\
\hline Ligand/ion & & & 25 & \\
\hline Water & 42 & 51 & 43 & 63 \\
\hline \multicolumn{5}{|l|}{ R.m.s deviations } \\
\hline Bond lengths $(\AA)$ & 0.011 & 0.015 & 0.015 & 0.015 \\
\hline Bond angles (deg) & 1.52 & 1.73 & 1.71 & 1.75 \\
\hline
\end{tabular}

${ }^{\mathrm{a}}$ Highest resolution shell is shown in parentheses.

${ }^{\mathrm{b}} R_{\text {merge }}=\sum_{h} \sum_{i}\left|I_{h i}-\left\langle I_{h}\right\rangle\right| / \sum_{h} \sum_{i} I_{h i}$.

${ }^{\mathrm{c}} R=\sum_{h}\left|F_{h}^{\mathrm{o}}-F_{h}^{\mathrm{c}}\right| / \sum_{h} F_{h}^{\mathrm{o}}$. 
space group $\mathrm{C} 2$, residues $1-54$ and 57-254 in chain $\mathrm{A}$ and 1-54, 58-96 and 102-254 in chain $B$ in space group $P 2{ }_{1} 2_{1} 2$, residues $1-254$ in chain A and 1-54 and 58-254 in chain B in space group R3. For the complex structure, the PrmA C-terminal domain and the L11 $\mathrm{N}$-terminal domain were located by molecular replacement. Partial structures were then built manually into $F_{\mathrm{o}}-F_{\mathrm{c}}$ difference density maps with the program $O$ (Jones et al, 1991), and the full-length domains were placed by least-squares alignment with the partial structures. The final complex model contains residues 1-55, 56-97 and 102-254 of PrmA and 1-139 of L11. The models were refined with Refmac (Murshudov et al, 1997), and the CCP4 package (Bailey, 1994) was used for subsequent calculations. The stereochemical quality of the models was assessed with PROCHECK (Laskowski et al, 1993).

The Ramachandran statistics (most favored/additionally allowed/generously allowed/disallowed) are 95.5/4.0/0.5/0.0\% for PrmA in space group C2, 89.9/9.3/0.8/0.0\% for PrmA in space group $\mathrm{P}_{1} 2_{1} 2,93.7 / 5.3 / 1.0 / 0.0 \%$ for PrmA in space group R3 and $84.3 / 15.1 / 0.6 / 0.0 \%$ for the PrmA-L11 complex. The structure refinement statistics are summarized in Table I.

Figures were generated using Pymol (http://pymol.sourceforge. net), Apbs (Baker et al, 2001), JalView (Clamp et al, 2004) and LigPlot (Wallace et al, 1995). Sequence alignments were generated with ClustalW (Thompson et al, 1994).

\section{References}

Agrawal RK, Linde J, Sengupta J, Nierhaus KH, Frank J (2001) Localization of L11 protein on the ribosome and elucidation of its involvement in EF-G-dependent translocation. J Mol Biol 311: $777-787$

Bailey S (1994) The Ccp4 suite-programs for protein crystallography. Acta Crystallogr D 50: 760-763

Baker NA, Sept D, Joseph S, Holst MJ, McCammon JA (2001) Electrostatics of nanosystems: application to microtubules and the ribosome. Proc Natl Acad Sci USA 98: 10037-10041

Cameron DM, Gregory ST, Thompson J, Suh MJ, Limbach PA, Dahlberg AE (2004a) Thermus thermophilus L11 methyltransferase, PrmA, is dispensable for growth and preferentially modifies free ribosomal protein L11 prior to ribosome assembly. J Bacteriol 186: $5819-5825$

Cameron DM, Thompson J, Gregory ST, March PE, Dahlberg AE (2004b) Thiostrepton-resistant mutants of Thermus thermophilus. Nucleic Acids Res 32: 3220-3227

Cameron DM, Thompson J, March PE, Dahlberg AE (2002) Initiation factor IF2, thiostrepton and micrococcin prevent the binding of elongation factor $\mathrm{G}$ to the Escherichia coli ribosome. J Mol Biol 319: 27-35

Clamp M, Cuff J, Searle SM, Barton GJ (2004) The Jalview Java alignment editor. Bioinformatics 20: 426-427

Colson C, Lhoest J, Urlings C (1979) Genetics of ribosomal-protein methylation in Escherichia coli. III. Map position of 2 genes, PrmA and PrmB, governing methylation of proteins L11 and L3. Mol Gen Genet 169: 245-250

Dincbas-Renqvist V, Engstrom A, Mora L, Heurgue-Hamard V, Buckingham R, Ehrenberg M (2000) A post-translational modification in the GGQ motif of RF2 from Escherichia coli stimulates termination of translation. EMBO J 19: 6900-6907

Dognin MJ, Wittmann-Liebold B (1980) Purification and primary structure determination of the N-terminal blocked protein, L11, from Escherichia coli ribosomes. Eur J Biochem 112: 131-151

Graille M, Heurgue-Hamard V, Champ S, Mora L, Scrima N, Ulryck N, van Tilbeurgh H, Buckingham RH (2005) Molecular basis for bacterial class I release factor methylation by PrmC. Mol Cell 20: 917-927

Heurgue-Hamard V, Champ S, Engstrom A, Ehrenberg M, Buckingham RH (2002) The hemK gene in Escherichia coli encodes the $\mathrm{N}$-5-glutamine methyltransferase that modifies peptide release factors. EMBO J 21: 769-778

Holm L, Sander C (1993) Protein-structure comparison by alignment of distance matrices. J Mol Biol 233: 123-138

Ilin S, Hoskin A, Ohlenschlager O, Jonker HRA, Schwalbe $\mathrm{H}$, Wohnert J (2005) Domain reorientation and induced fit upon RNA binding: solution structure and dynamics of ribosomal

\section{Accession codes}

The atomic coordinates and structure factors have been deposited in the Protein Data Bank with accession codes 2NXC for PrmA in space group $\mathrm{C} 2,2 \mathrm{NXJ}$ for PrmA in space group $\mathrm{P} 2{ }_{1} 2{ }_{1} 2,2 \mathrm{NXE}$ for PrmA in space group R3 and 2NXN for the PrmA-L11 complex structure.

\section{Supplementary data}

Supplementary data are available at The EMBO Journal Online (http://www.embojournal.org).

\section{Acknowledgements}

We thank Jill Thompson for useful discussions, Theodora Choli Papadopoulou for the L11 plasmid and Jean-Hervé Alix for the E. coli KNOK16 strain. We thank John Schwanof and Randy Abramowitz for access to the $\mathrm{X} 4 \mathrm{~A}$ and $\mathrm{X} 4 \mathrm{C}$ beamlines at the National Synchrotron Light Source and William Holmes and Hua Li for help with data collection at the synchrotron. This work was supported by grant GM19756 from the US National Institutes of Health to AED and by Brown University (to GJ).

protein L11 from Thermotoga maritima. Chembiochem 6: $1611-1618$

Jogl G, Tao X, Xu YW, Tong L (2001) COMO: a program for combined molecular replacement. Acta Crystallogr D 57: $1127-1134$

Jones TA, Zou JY, Cowan SW, Kjeldgaard M (1991) Improved methods for building protein models in electron-density maps and the location of errors in these models. Acta Crystallogr A 47: 110-119

Kaminishi T, Sakai H, Takemoto-Hori C, Terada T, Nakagawa N, Maoka N, Kuramitsu S, Shirouzu M, Yokoyama S (2003) Crystallization and preliminary X-ray diffraction analysis of ribosomal protein L11 methyltransferase from Thermus thermophilus HB8. Acta Crystallogr D 59: 930-932

Kozbial PZ, Mushegian AR (2005) Natural history of S-adenosylmethionine-binding proteins. Bmc Struct Biol 5: 9

Kukimoto-Niino M, Murayama K, Inoue M, Terada T, Tame JRH, Kuramitsu S, Shirouzu M, Yokoyama S (2004) Crystal structure of the GTP-binding protein Obg from Thermus thermophilus HB8. J Mol Biol 337: 761-770

Laskowski RA, Macarthur MW, Moss DS, Thornton JM (1993) Procheck-a program to check the stereochemical quality of protein structures. J Appl Crystallogr 26: 283-291

Limbach PA, Crain PF, McCloskey JA (1994) Summary: the modified nucleosides of RNA. Nucleic Acids Res 22: 2183-2196

Martin JL, McMillan FM (2002) SAM (dependent) I AM: the $S$-adenosylmethionine-dependent methyltransferase fold. Curr Opin Struct Biol 12: 783-793

McCoy AJ, Grosse-Kunstleve RW, Storoni LC, Read RJ (2005) Likelihood-enhanced fast translation functions. Acta Crystallogr D 61: 458-464

Miller JH (1992) A Short Course in Bacterial Genetics. Plainview, NY: Cold Spring Harbor Laboratory Press

Murshudov GN, Vagin AA, Dodson EJ (1997) Refinement of macromolecular structures by the maximum-likelihood method. Acta Crystallogr D 53: 240-255

Otwinowski Z, Minor W (1997) Processing of X-ray diffraction data collected in oscillation mode. Methods Enzymol 276: 307-326

Rohl R, Nierhaus KH (1979) Methyl-groups of ribosomal-protein L11 are not related to the synthesis of ppGpp. Mol Gen Genet 170: 187-189

Schubert HL, Blumenthal RM, Cheng XD (2003a) Many paths to methyltransfer: a chronicle of convergence. Trends Biochem Sci 28: $329-335$

Schubert HL, Phillips JD, Hill CP (2003b) Structures along the catalytic pathway of $\mathrm{PrmC} / \mathrm{HemK}$, an $\mathrm{N}$-5-glutamine AdoMetdependent methyltransferase. Biochemistry 42: 5592-5599 
Stark H, Rodnina MV, Wieden HJ, Zemlin F, Wintermeyer W, van Heel M (2002) Ribosome interactions of aminoacyl-tRNA and elongation factor Tu in the codon-recognition complex. Nat Struct Biol 9: 849-854

Thompson JD, Higgins DG, Gibson TJ (1994) Clustal-W-improving the sensitivity of progressive multiple sequence alignment through sequence weighting, position-specific gap penalties and weight matrix choice. Nucleic Acids Res 22: 4673-4680

Trievel RC (2004) Structure and function of histone methyltransferases. Crit Rev Eukaryot Gene Expr 14: 147-169

Valle M, Zavialov A, Li W, Stagg SM, Sengupta J, Nielsen RC, Nissen P, Harvey SC, Ehrenberg M, Frank J (2003) Incorporation of aminoacyl-tRNA into the ribosome as seen by cryo-electron microscopy (vol 10, pg 899, 2003). Nat Struct Biol 10: 1074

Vanet A, Plumbridge JA, Alix JH (1993) Cotranscription of 2 genes necessary for ribosomal protein-L11 methylation (PrmA) and pantothenate transport (PanF) in Escherichia coli K-12. J Bacteriol 175: 7178-7188

Vanet A, Plumbridge JA, Guerin MF, Alix JH (1994) Ribosomalprotein methylation in Escherichia coli-the gene PrmA, encoding the ribosomal-protein L11 methyltransferase, is dispensable. Mol Microbiol 14: 947-958

Vannoort JM, Kraal B, Sinjorgo KMC, Persoon NLM, Johanns ESD, Bosch L (1986) Methylation in vivo of elongation-factor EF-Tu at Lysine-56 decreases the rate of transfer RNA-dependent GTP hydrolysis. Eur J Biochem 160: 557-561

Wallace AC, Laskowski RA, Thornton JM (1995) Ligplot-a program to generate schematic diagrams of protein ligand interactions. Protein Eng 8: 127-134

Wimberly BT, Guymon R, McCutcheon JP, White SW Ramakrishnan V (1999) A detailed view of a ribosomal active site: the structure of the L11-RNA complex. Cell 97: 491-502

Xiao B, Jing C, Wilson JR, Walker PA, Vasisht N, Kelly G, Howell S, Taylor IA, Blackburn GM, Gamblin SJ (2003) Structure and catalytic mechanism of the human histone methyltransferase SET7/9. Nature 421: 652-656

Zhang X, Yang Z, Khan SI, Horton JR, Tamaru H, Selker EU, Cheng $\mathrm{XD}$ (2003) Structural basis for the product specificity of histone lysine methyltransferases. Mol Cell 12: 177-185 